\title{
Roald Dahl auteur-illustrateur : de l'image au texte, les débuts de la genèse de Fantastic Mr Fox
}

\section{Christine Collière-Whiteside}

\section{(2) OpenEdition}

1 Journals

\section{Édition électronique}

URL : http://journals.openedition.org/genesis/4044

DOI : 10.4000/genesis.4044

ISSN : 2268-1590

Éditeur :

Presses universitaires de Paris Sorbonne (PUPS), Société internationale de génétique artistique littéraire et scientifique (SIGALES)

Édition imprimée

Date de publication : 1 juin 2019

Pagination : 69-80

ISSN : 1167-5101

Référence électronique

Christine Collière-Whiteside, "Roald Dahl auteur-illustrateur : de l'image au texte, les débuts de la genèse de Fantastic Mr Fox », Genesis [En ligne], 48 | 2019, mis en ligne le 01 juin 2020, consulté le 25 janvier 2021. URL : http://journals.openedition.org/genesis/4044; DOI : https://doi.org/10.4000/ genesis. 4044 


\title{
Roald Dahl auteur-illustrateur : de l'image au texte, les débuts de la genèse de Fantastic Mr Fox
}

\author{
Christine Collière-Whiteside
}

$\mathrm{E}$ n 1968, Roald Dahl se lance dans l'écriture d'une nouvelle histoire pour enfants, destinée à des lecteurs débutants, bien plus jeunes que ceux de ses œuvres précédentes, James and the Giant Peach (1961), Charlie and the Chocolate Factory (1964) et The Magic Finger (1966). Il conçoit donc son nouveau livre comme un album, et au lieu d'écrire d'abord le texte pour le donner ensuite à illustrer à un artiste, il s'essaie à l'invention conjointe du texte et des images, sans avoir nécessairement eu l'intention de publier ses propres illustrations. Les deux manuscrits illustrés qui en résultent constituent une occurrence unique dans les archives de Roald Dahl, conservées au Roald Dahl Museum and Story Center à Great Missenden. Au cours des campagnes de révision successives, on voit le projet évoluer, le texte s'allonger et l'œuvre changer de nature pour devenir, non plus un album, mais un roman illustré, quoique beaucoup plus court que James ou Charlie. Il sera publié en 1970 sous le titre Fantastic Mr Fox ${ }^{1}$ avec des illustrations de Donald Chaffin.

On peut s'interroger sur le statut de cette expérience unique : constitue-t-elle un révélateur de mécanismes inhérents à la création chez Dahl, ou ne faut-il y voir que l'expérimentation d'un esprit plein de curiosité? L'intention première, écrire un album pour de très jeunes enfants, suffitelle à expliquer que Dahl ait travaillé conjointement sur le texte et les images de cette œuvre? Il était, comme son père, collectionneur et marchand d'art et d'antiquités. Ses croquis montrent qu'il avait une certaine habileté au dessin. Dès la publication de James, fort de ses contacts dans le monde artistique, il s'était impliqué dans le choix des illustrateurs de ses romans pour enfants et s'intéressait toujours de près à leur travail, même si ce n'est qu'à partir de 1978 qu'une véritable collaboration s'est installée avec Quentin Blake. Il ne semble cependant jamais avoir eu de velléités artistiques (contrairement à Lewis Carroll par exemple, qui avait initialement l'intention de publier Alice avec ses propres illustrations). Dans ce contexte, le passage par l'image a-t-il infléchi l'œuvre et, le cas échéant, de quelle façon? Nous faisons l'hypothèse que l'étude génétique des brouillons de $M r$ Fox fait émerger des préoccupations communes à ce roman et aux autres textes de Dahl : d'une part la thématique du vol qui irrigue toute l'œuvre de l'auteur, ancien pilote, d'autre part la réflexion sur la nature humaine qui sera plus tard développée dans The $B F G^{2}$.

Les romans de Dahl pour la jeunesse ont généralement pour héros principal un enfant, parfois accompagné d'un équipage d'animaux plus ou moins anthropomorphiques, comme les insectes qui accompagnent James dans la pêche géante, ou le cygne de Billy dans The Minpins. Fantastic Mr Fox est la première œuvre de Dahl dont le héros soit un animal, un choix caractéristique des œuvres qu'il destinait à un lectorat très jeune, comme The Enormous Crocodile et The Giraffe and the Pelly and Me. Trois fermiers aux personnalités très différentes, Boggis, Bunce et Bean, s'allient pour se débarrasser du rusé Mr Fox qui les vole régulièrement et de sa famille. Ils réussissent d'abord à mutiler Mr Fox, puis détruisent son terrier à l'aide de puissantes tractopelles, tandis que les renards creusent de plus en plus profondément pour leur échapper. Quand les fermiers épuisés changent de stratégie et décident de les affamer, Mr Fox creuse un tunnel menant directement sous le poulailler de Boggis, puis sous les garde-manger des deux autres fermiers. Tandis que les fermiers montent toujours la garde, il organise un grand

\footnotetext{
1. Fantastic Mr Fox, ill. Donald Chaffin, Londres, George Allen \& Unwin et New York, Alfred A. Knopf, 1970.

2. Voir le chapitre «Journey to Dream Country» : «Human beans $[$ sic $]$ is the only animals that is killing their own kind. » [Les hommes de terre sont les seuls animaux qui tuent les membres de leur propre espèce.], The BFG, Puffin Books 2001, p. 70.
} 
banquet de victoire auquel sont conviés les autres animaux dont l'habitat a été détruit et décide de ne plus jamais sortir à l'air libre et de creuser tout un village souterrain.

\section{Le dossier génétique}

Le dossier génétique de Fantastic Mr Fox (RD/2/93) est constitué de treize documents au total. Les quatre premières versions, toutes intitulées «Mr Fox», correspondent à la phase durant laquelle le livre est conçu comme un album, et présentent une alternance de texte quasi seul et de texte illustré. La première (RD/2/9/1), désignée comme «First Draft» par l'auteur, est une liasse de 46 feuilles volantes manuscrites, écrites au crayon sur le papier jaune ligné des blocs-notes américains dits «legal pads ${ }^{4}$ » typique des avant-textes de Dahl. L'auteur n'a écrit que dans la moitié inférieure de chaque page, au recto uniquement, la moitié supérieure étant prévue pour des illustrations, qui sont présentes sur les trois quarts des pages environ. Le texte a donc probablement été recopié5 à partir d'une version précédente, la segmentation du texte en pages ayant été faite lors de la copie. Chaque croquis constitue une illustration proprement dite, quoique rapidement esquissée, voire complétée par des explications, par exemple, sur la figure 1, les mots «town» et «wood» et la note «(cross-section of town and wood, showing underground tunnel)». Ce ne sont pas des études laissant coexister plusieurs possibilités, ni des essais témoignant de recherches concernant les personnages ou l'intrigue, ce qui laisse penser que le texte a dû généralement être écrit d'abord, puis illustré. Si l'invention venait à Dahl en dessinant, les brouillons qui portaient la trace de ces griffonnages ont disparu.

Ce premier brouillon a été ensuite tapé à la machine (par Dahl lui-même ou par un secrétaire) en double exemplaire avec une copie carbone selon une mise en page particulière permettant une découpe en petits rectangles comportant une à quatorze courtes lignes de texte. La copie principale a ainsi été fractionnée, et on en trouve les morceaux fixés avec du scotch sur les feuilles blanches du second document (RD/2/9/2), un livre relié à couverture blanche, constituant une première maquette de l'album, où Dahl a dessiné au crayon sur toutes les pages les esquisses des illustrations. Dans cette première maquette, les illustrations s'étendent parfois sur une double page, parfois sur une seule page. La disposition du texte sur les pages n'a pas fait l'objet d'un travail particulièrement élaboré : un seul bloc de texte par page, toujours de la même largeur', placé en bas, sous les illustrations ${ }^{7}$ si bien que la seule contrainte lors de la copie à la machine était de respecter une largeur maximale.

Le document suivant (RD/2/9/3), dit «Second Draft», est une liasse d'une trentaine de pages, utilisées seulement au recto, sur lesquelles ont été scotchés des morceaux de la copie carbone du tapuscrit que nous venons de décrire, utilisé pour la première maquette. Cette version ne comporte que du texte, soit manuscrit, au crayon sur les feuilles jaunes, soit tapuscrit sur les coupures de papier blanc. Aucun espace n'est prévu cette fois pour un travail sur des esquisses, le texte commençant toujours vers le haut de la page et chaque feuillet correspondant non plus à une page mais à une double page de la maquette.

Le quatrième document $(\mathrm{RD} / 2 / 9 / 4)$ est une seconde maquette, fabriquée selon le même procédé que la première : le deuxième brouillon a été tapé au propre, le tapuscrit résultant a été découpé, les morceaux ont été scotchés dans un second volume relié et illustrés au crayon par l'auteur. Une note de la main de Dahl sur la première page illustrée indique que toutes les illustrations sont en double page, ce qui confirme que l'auteur envisage bien l'œuvre comme un album. La copie carbone de ce deuxième tapuscrit $(\mathrm{RD} / 2 / 9 / 5)$ porte des corrections ultérieures et représente donc à la fois une étape intermédiaire entre le deuxième brouillon et la seconde maquette si on ignore les corrections,

3. J'adresse mes remerciements les plus chaleureux à Rachel White des archives Dahl à Great Missenden pour avoir attiré mon attention sur ce dossier.

4. Le terme «legal» désigne un format de papier américain $(216 \mathrm{~mm} \times 355,6 \mathrm{~mm})$, très proche du format A4. Dahl était très attaché à ses outils d'écriture et faisait venir spécialement ses blocs-notes et ses crayons Dixon Ticonderoga des États-Unis par ses éditeurs américains.

5. Des traces de révision apparaissent sur plusieurs pages, indiquant que le texte a été retravaillé au cours de la copie.

6. À l'exception des premières pages où des renvois à la ligne donnent un rythme particulier au texte et raccourcissent encore la largeur des morceaux.

7. Seule l'illustration particulière de la double page représentant la rue en perspective ( «If you had been able to see under the street from above, this is what it would have looked like») a obligé Dahl à coller cette phrase en haut de la page, dans l'espace libre (voir fig. 5). 
et l'étape suivant la seconde maquette si on tient compte des corrections.

Roald Dahl envoya alors son manuscrit chez son éditeur du moment, Random House. Après une série d'échanges avec les éditeurs, au cours desquels il faut supposer l'existence d'une ou plusieurs versions intermédiaires, aujourd'hui perdues, le texte, désormais intitulé «Boggis and Bunce and Bean» (RD/2/9/6), s'est considérablement allongé. Il s'agit d'un manuscrit au crayon sur papier jaune d'une soixantaine de pages, comportant quelques croquis mais essentiellement constitué de texte couvrant l'intégralité des rectos, et divisé en chapitres. Le livre n'était plus destiné à des lecteurs débutants, mais plutôt à la même tranche d'âge que les œuvres précédentes de Dahl. Le statut de l'œuvre avait changé avec la place dévolue aux illustrations, il s'agissait désormais d'un «chapter book» illustré.

\section{De l'album au livre illustré : le rôle des éditeurs}

Dahl tenait en très haute estime les livres écrits pour de très jeunes enfants. Il était très fier de The Enormous Crocodile qu'il publia par la suite, en 1978. Il exprima cette opinion à plusieurs reprises, notamment dans une lettre de 1985 : «Pour moi, écrire le texte d'un très bon album, court, pour de jeunes enfants est ce qu'il y a de plus difficile, plus difficile qu'un roman, une nouvelle ou un roman pour enfants ${ }^{8}$. » Il donne deux raisons à cela : il faut d'abord arriver à capter l'attention d'un très jeune enfant : «En seulement cinq mille mots environ, il faut bâtir une intrigue complète et équilibrée, qui soit si captivante, si palpitante, si amusante et si simple que l'enfant voudra l'entendre encore et encore.» Secundo, «il est nécessaire que l'histoire se prête parfaitement à être illustrée».

La maquette illustrée, seconde version de «Mr Fox», que Dahl envoya à son éditeur, ne remplissait qu'une seule de ces deux conditions : l'intrigue est en effet trop simple. L'histoire commence in medias res par un siège : des «fermiers » essaient de déloger la famille renard de sa tanière soit en creusant soit en l'affamant, sans qu'aucune justification à cet acharnement (ils utilisent d'énormes machines) ne soit donnée. Le nombre et le nom des fermiers restent inconnus, ils ne sont jamais décrits et n'inspirent pas d'antipathie particulière au lecteur. Mr Fox a alors simplement l'idée de creuser un tunnel jusqu'au village voisin (voir fig. 1) afin de pouvoir voler de la nourriture dans les magasins de la grand-rue. Le succès de ce projet conclut l'histoire. La première version de l'intrigue était encore moins captivante, car au lieu du suspense et de l'excitation de l'affrontement initial entre fermiers et renards essayant de creuser plus vite les uns que les autres, c'était simplement les aspirations bourgeoises de Mrs Fox qui servaient de déclencheur (voir fig. 8). Elle rêve en effet d'une maison avec tout le confort moderne : une cuisine avec un beau four et une panoplie complète de casseroles et de marmites, une belle salle à manger avec une table bien garnie ${ }^{9}$, autant de biens de consommation que Mr Fox et ses fils s'empressent alors de voler dans les magasins après avoir creusé tout un réseau de galeries sous les rues, ainsi qu'un réfrigérateur, des tapis, des lampes et toutes sortes de meubles.

Sans surprise, les éditeurs ne furent pas convaincus. Plutôt que de critiquer l'intrigue, au risque de vexer l'auteur qu'ils savaient capables de donner son manuscrit à une maison d'édition concurrente, ils pointèrent du doigt la moralité douteuse de l'histoire, qui pouvait être considérée comme une apologie du vol10. Un jeune rédacteur en chef, Fabio Coen, proposa alors à Dahl un nouveau scénario dans lequel ce seraient les fermiers, au nombre de trois, qui

8. «Writing the text of a short first-rate illustrated book for young children is the most difficult task of all, more difficult than a novel, a short story, or a full length children's book. [...] Within the span of approximately five thousand words one must construct a complete and rounded plot that is so absorbing, exciting, funny and simple that the child will want to hear it over again and again. [...] The story must lend itself marvelously to illustration. » Lettre du 25 juin 1985, écrite pour «le bulletin d'information de Tom Maschler», (RD/6/2/1/21), citée avec l'aimable autorisation de Roald Dahl Story Company Ltd.

9. «Just look at that fine kitchen! Look at that pretty oven! Look at those lovely pots and pans!... Just look at that fine dining-room! Look at that pretty table! Look at that lovely food!», «Mr Fox» (RD/2/9/2), Maquette 1.

10. Donald Sturrock, Storyteller : the Life of Roald Dahl, London, HarperPress, 2010, p. 444-446. On notera que Sturrock se trompe lorsqu'il ajoute à ces critiques des réserves concernant des allusions à la guerre mal venues selon les éditeurs de Random House dans un contexte de contestation croissante des interventions militaires américaines au Viet Nam. En réalité, ces réserves concernent une version ultérieure du roman, le passage que cite Sturrock se trouvant dans «Boggis and Bunce and Bean» (RD/2/9/6), fol. 46. Les modifications proposées par les éditeurs en novembre 1968 cherchent donc seulement à remédier à la pauvreté de l'intrigue et à la moralité douteuse de l'histoire. 


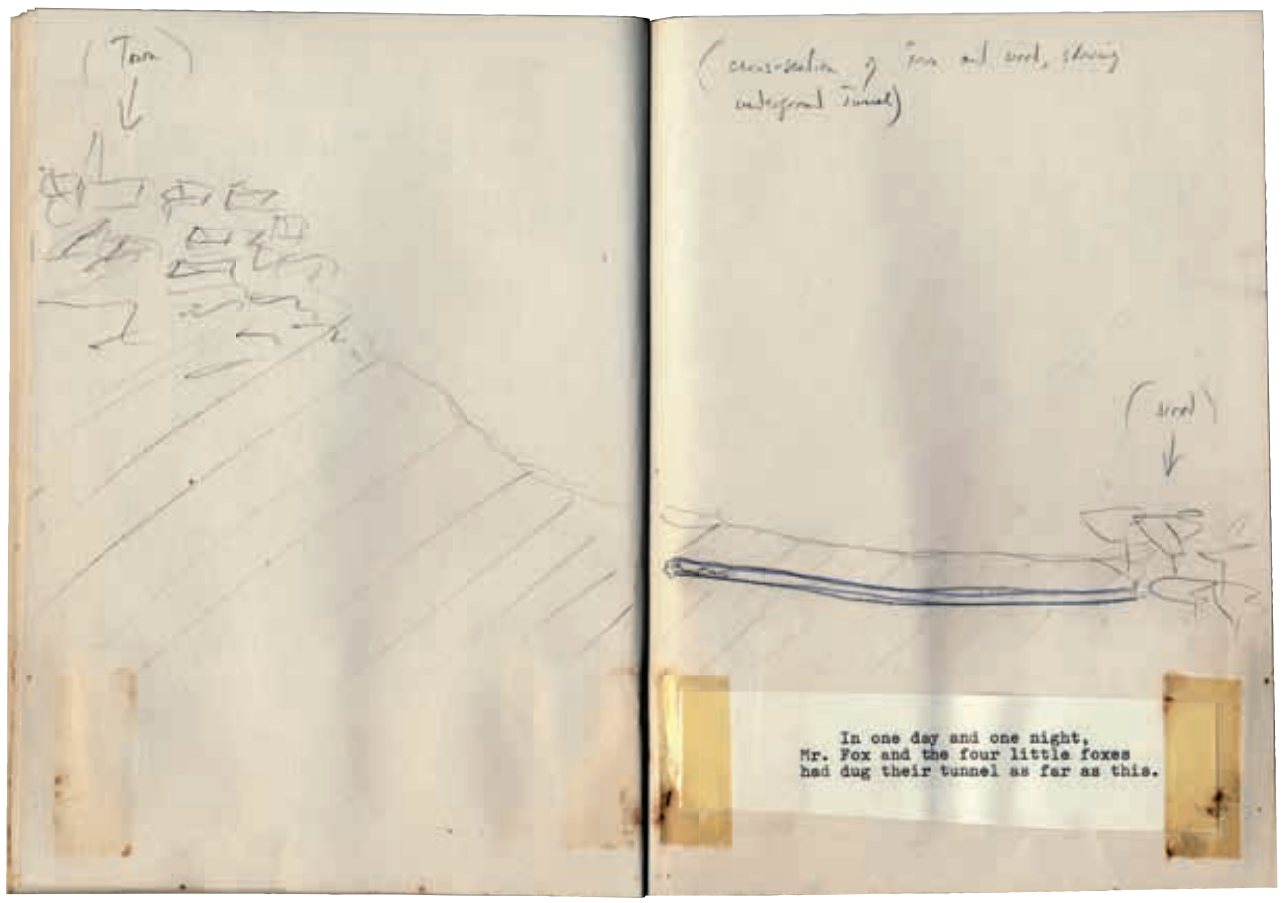

Fig. 1 : «In one day and one night», «Mr Fox », Maquette $1(\mathrm{RD} / 2 / 9 / 2)$, double page, pas de pagination.

() Roald Dahl Story Company Ltd

seraient victimes des vols. La proposition plut à Dahl qui adopta les suggestions de Coen jusque dans les moindres détails, déclarant : "Vous me faites des suggestions si pertinentes que j'ai l'impression de commettre un plagiat en les acceptant ${ }^{11}$.» Le nouveau titre, «Boggis and Bunce and Bean», place effectivement les trois fermiers au centre de l'histoire, qui s'ouvre désormais sur la description des trois fermes au lieu de la tanière des renards, et qui inclut deux étapes où sont explicitées les raisons pour lesquelles les renards ont faim et les motifs de la haine des fermiers. Dahl a également adopté certains détails presque à la lettre : Coen suggérait que l'un des fermiers élève des poulets, que le second produise du porc et le troisième du cidre ${ }^{12}$. Dans «Boggis and Bunce and Bean», comme dans la version publiée, le premier fermier produit des poules, le second des canards et des oies, et le dernier des dindes et du cidre, boisson que le cadet des renardeaux apprécie particulièrement, comme le suggérait aussi Coen dans sa lettre.

Fabio Coen a ainsi joué un rôle décisif dans la genèse de Fantastic Mr Fox, comme l'avait fait avant lui le premier agent littéraire de Dahl, Sheila St Lawrence, pour la genèse de James ${ }^{13}$. Si le terme de «plagiat» employé par Dahl est une exagération, il témoigne de l'importance des collaborations de l'auteur avec ses nombreux éditeurs britanniques et américains dans la genèse de ses œuvres. Pourtant Coen ne suggérait en aucune façon d'abandonner l'idée d'un album pour lecteurs débutants et son format court, très illustré. Le scénario qu'il proposait à Dahl n'appelait pas nécessairement une expansion du texte et Coen indiquait d'ailleurs qu'il trouvait l'histoire trop longue. En provoquant une modification de l'intrigue et des personnages, en fournissant au héros la motivation et les antagonistes qui lui manquaient, Coen a relancé l'écriture dans une direction différente qui donne plus de profondeur aux personnages. La pauvreté de l'intrigue initiale peut s'expliquer par un effort de simplification pour un lectorat nouveau pour l'auteur. En déterminant le nombre des fermiers, Coen permet à Dahl de développer l'histoire selon une structure répétitive : Mr Fox rend successivement visite à chacune des fermes, et chaque visite permet d'étoffer progressivement le personnage de Mr Fox et de lui construire une nouvelle place non seulement au sein de sa famille mais au centre d'une communauté d'animaux divers, tous conviés au banquet final durant lequel Mr Fox livre sa philosophie.

11. «You have come up with suggestions so good that I feel almost as though I am committing plagiarism in accepting them. » Lettre à Fabio Coen du 25 novembre 1968, citée dans Storyteller, p. 446.

12. Lettre de Fabio Coen du 20 novembre 1968, conservée au Roald Dahl Museum and Story Center, (RD/1/4/2/1/1 et 2), citée avec l'aimable autorisation de la Roald Dahl Story Company Ltd.

13. Donald Sturrock raconte en détail les relations tumultueuses de Dahl avec ses agents littéraires et ses éditeurs, alternant des périodes d'enthousiasme et de gratitude pour leurs suggestions et leur collaboration et des séparations suite à de féroces querelles. Voir par exemple les pages 378-379 ou 502-509 de Storyteller. Ces collaborations sont une constante de la carrière littéraire de Dahl et témoignent de la complexité des relations entre un auteur et les différents acteurs d'une genèse, qui sont explorées dans l'article de Nicolas Donin et Daniel Ferrer «Auteur(s) et acteurs de la genèse », Genesis, n 41, «Créer à plusieurs mains », 2015, p. 7-26. 


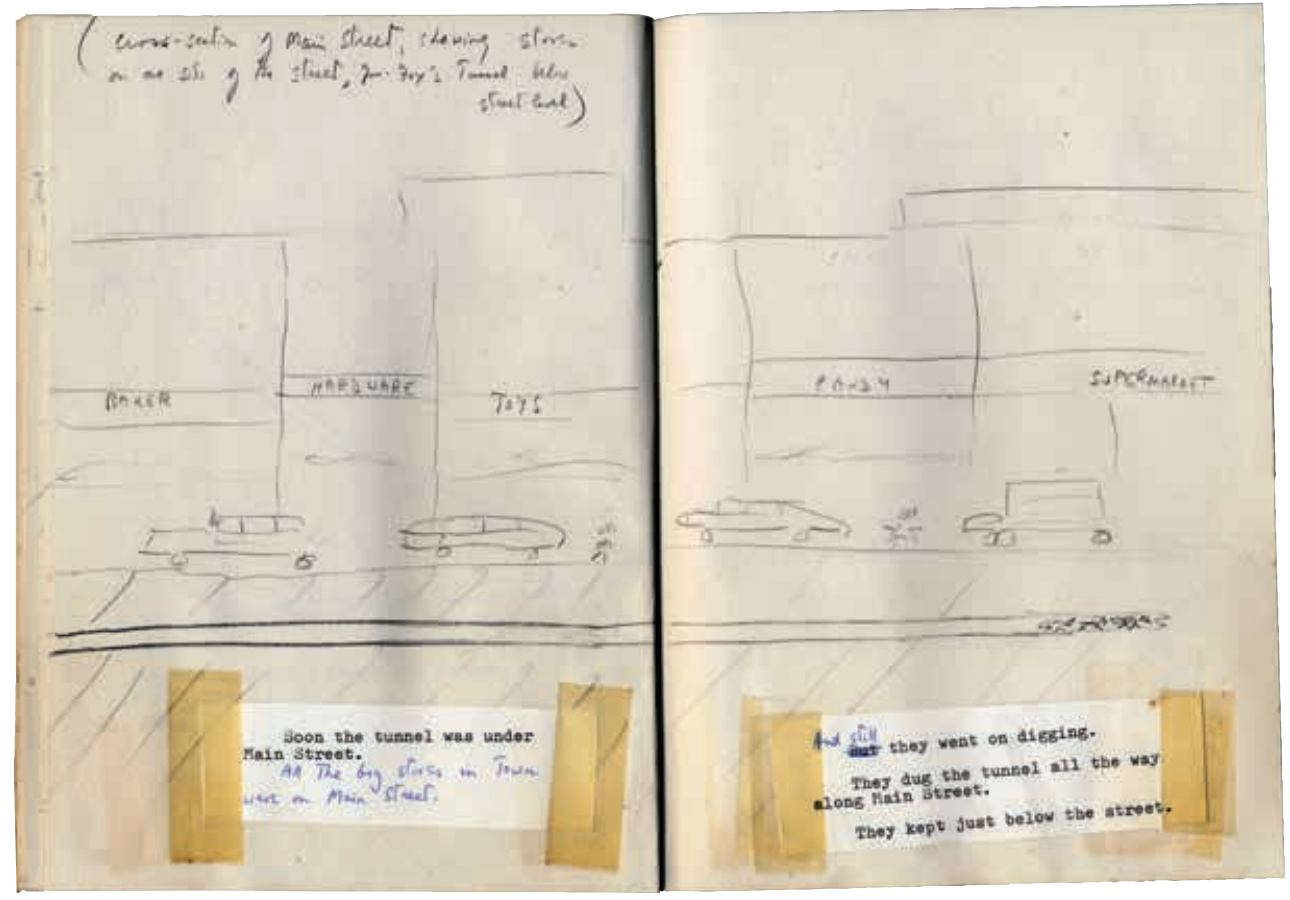

Fig. 2 «Soon the tunnel was under Main Street», «Mr Fox», Maquette 1 (RD/2/9/2), double page, sans pagination. (c) Roald Dahl Story Company Ltd

\section{Des images au texte : l'écriture et le vol}

Si l'intrigue initiale était faible, les deux premières versions de l'histoire se prêtaient en revanche merveilleusement bien à l'illustration, remplissant la première condition définie par Dahl. Alors que dans ses autres œuvres Dahl a recours au fantastique pour créer des images inoubliables, à commencer par la pêche géante de James flottant dans les airs au-dessus de l'Atlantique, il n'y a rien de contraire aux lois du monde naturel dans Fantastic Mr Fox, sinon l'exagération de la vitesse à laquelle ils sont capables de creuser la terre, aussi rapidement que les machines. Quelque chose d'extraordinaire se dégage pourtant des images présentes dans les premières versions, en particulier lorsqu' on les voit telles que Dahl les avait imaginées, en découvrant les croquis des deux maquettes. En effet, ceux-ci représentent pour la plupart le paysage en coupe, montrant à la fois l'espace souterrain et ce qui se trouve à la surface afin que l'on voie la progression du tunnel (voir fig. 1) ou la position des renards juste sous la grand-rue et ses magasins (voir fig. 2), représentant ainsi visuellement le principe structurant de l'histoire, c'est-à-dire le contraste entre les ruses invisibles du renard, dont le lecteur connaît le secret, et l'inconscience des villageois vaquant à leurs occupations.

De «Mr Fox» à «Boggis and Bunce and Bean», on passe ainsi d'une œuvre déterminée par ce qu'elle convoquait visuellement à une histoire motivée par la construction d'une intrigue cohérente et le développement psychologique des personnages. Ainsi, dans la version finale, les personnages sont moins caricaturaux, plus complexes : les trois fermiers ont chacun une personnalité distincte et leurs relations reflètent les différents rapports de force qui les lient; Mr Fox subit un traumatisme au tout début de l'histoire lorsqu'il perd le beau panache qui faisait sa fierté («"Elle ne repoussera jamais", dit Maître Renard, très abattu. "Je resterai mutilé toute ma vie." $14 »$ ) tandis que Blaireau soulève un dilemme lorsqu'il s'interroge sur la moralité des vols commis par Mr Fox et lui-même (chapitre 14).

La comparaison entre les esquisses de Dahl pour «Mr Fox» et les illustrations publiées dans les éditions successives de Fantastic Mr Fox est révélatrice de cette évolution. Les deux premiers illustrateurs, Donald Chaffin et Jill Bennett, tout comme Quentin Blake plus tard, s'attachèrent surtout à dépeindre les personnages et non la disposition des paysages et des galeries souterraines. Ils ne choisirent presque jamais de représenter le paysage en coupe, à l'exception d'un unique dessin de Jill Bennett 15 (voir fig. 3). Dans leurs illustrations, le spectateur partage le point de vue des renards, à l'intérieur des galeries, participant intimement à leurs aventures et leurs peurs, comme dans le dessin inquiétant de Quentin Blake où l'on voit une pelle émerger du plafond (voir fig. 4). Dans les croquis de Dahl, le regard est plus détaché, comme celui d'un œil

14. . «"It will never grow again", said Mr Fox. "I shall be tail-less for the rest of my life." He looked very glum. » Fantastic Mr Fox, London, Puffin Books, 2013, chapitre 4, p. 5.

15. Fantastic Mr Fox, ill. Jill Bennett, London, Puffin Books, 1974. 


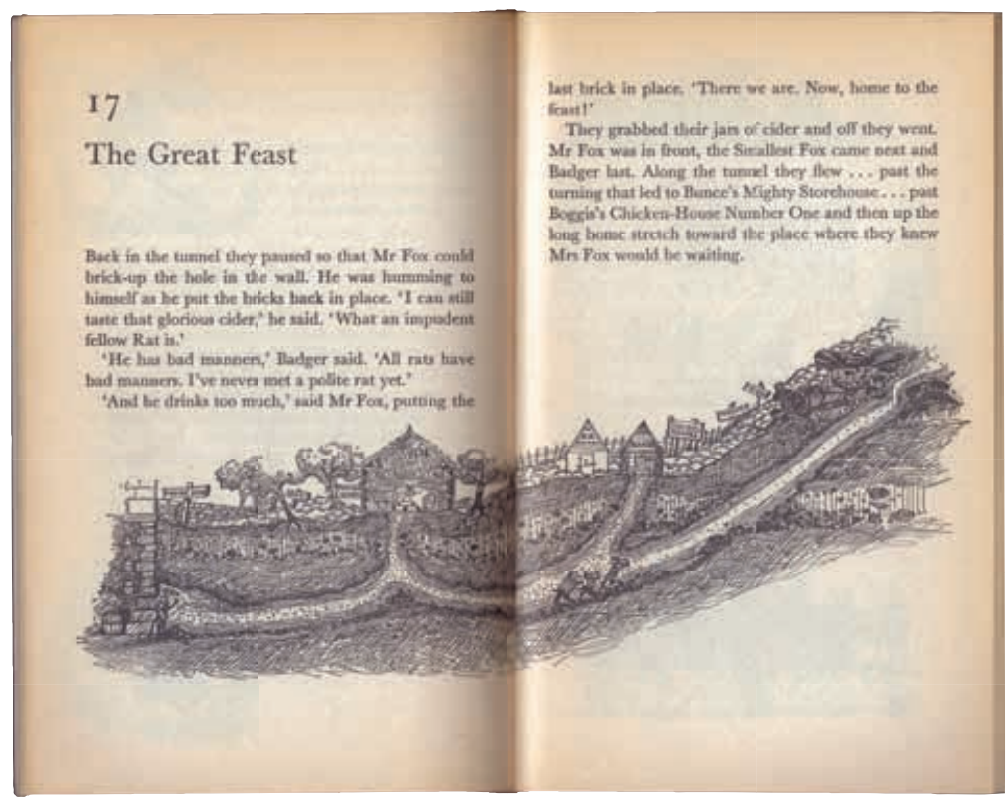

Fig. 3 : Fantastic Mr Fox, ill. Jill Bennett, London, Puffin Books, 1974, p. 78-79, reproduite avec l'aimable autorisation de l'artiste.

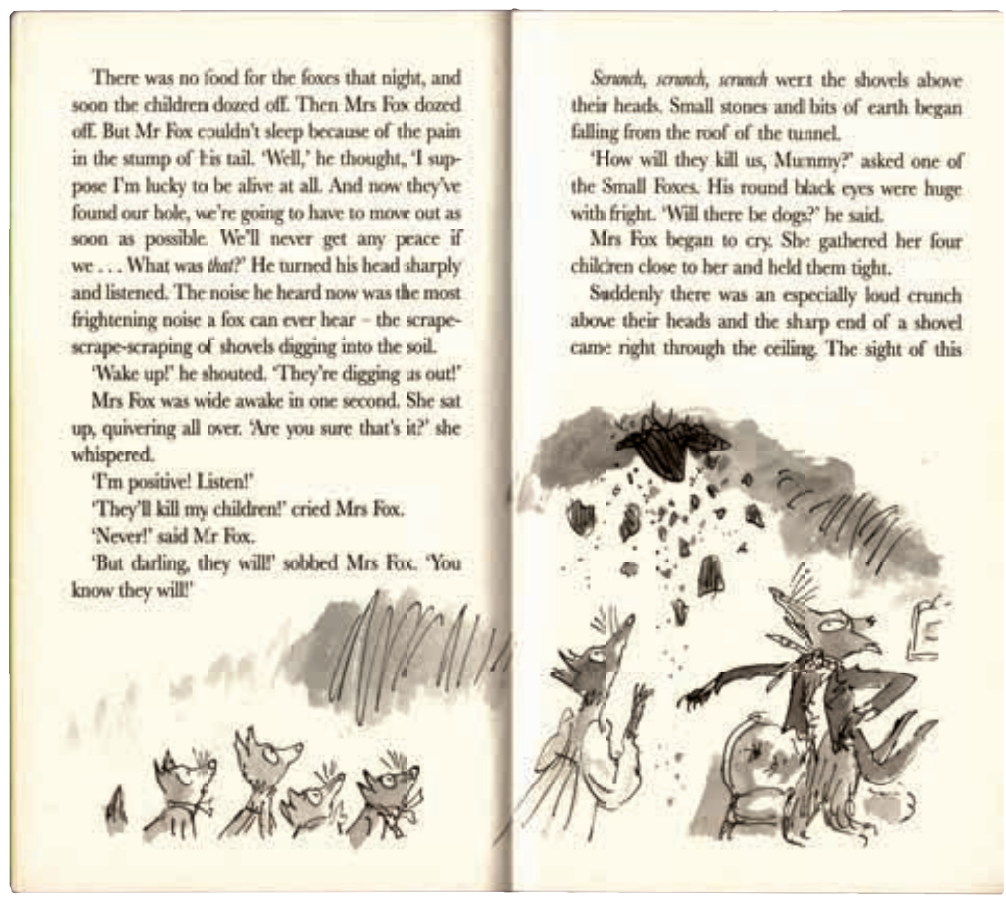

Fig. 4 : Fantastic Mr Fox, ill. Quentin Blake, London, Puffin Books, 2013, p. 16-17.

extérieur observant froidement les animaux, à la manière d'un scientifique observant une fourmilière dans un terrarium. Ce sentiment s'explique peut-être par la nature de ses illustrations, qui ne sont que des croquis (voir fig. 2), mais le texte lui-même donne peu de profondeur psychologique aux personnages.
Dans ces premières versions, les dessins de Dahl relèvent presque du travail de l'architecte (voir fig. 3), comme ce croquis particulièrement saisissant où la rue et les galeries sous-jacentes ne sont plus représentées en coupe mais vues de dessus (voir fig. 5), ce qui ouvre véritablement un espace tridimensionnel. On y voit d'abord, occupant toute la page, 


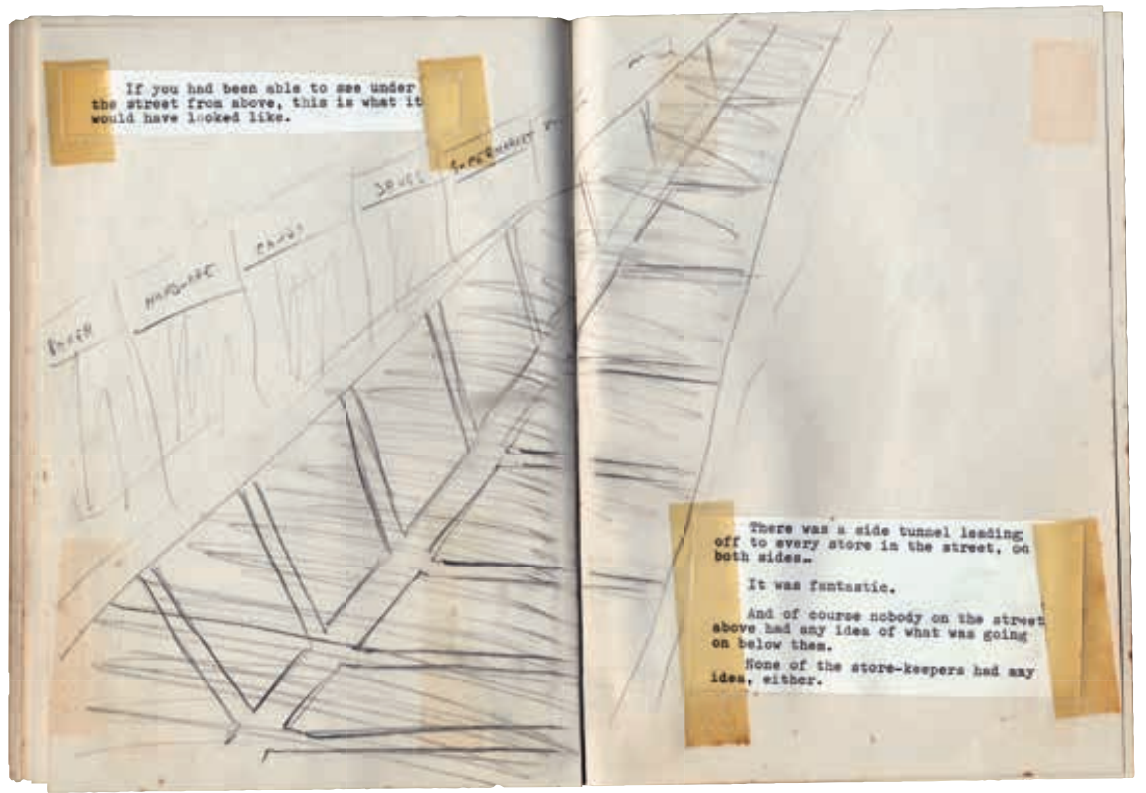

Fig. 5 «If you had been able to see under the street from above», «Mr Fox», Maquette 1 $(\mathrm{RD} / 2 / 9 / 2)$, double page, pas de pagination.

(c) Roald Dahl Story Company Ltd la galerie principale et les galeries latérales, dessinées à traits très marqués, beaucoup plus foncés que sur les autres pages. Les hachures représentent la surface du sol qui sépare l'espace souterrain de l'espace aérien tout en permettant de voir par transparence les galeries, tandis que se trouvent sur le côté les magasins qui sont supposés être verticaux. Ce tunnel qui s'étend à l'infini vers le point de fuite, presque comme une piste de décollage, suggère la liberté totale que ces galeries accordent aux renards, liberté dont ils peuvent profiter en toute impunité, comme le souligne le texte qui l'accompagne : «Bien sûr, personne dans la rue au-dessus n'avait la moindre idée de ce qui se passait sous leurs pieds ${ }^{16}$.» Un autre commentaire s'adresse au lecteur et lui donne par ailleurs le pouvoir de voir à travers la matière solide, faisant de lui un spectateur omniscient, contrairement aux habitants du village : «Si vous aviez été capable de voir sous la rue par en dessus, voici ce à quoi cela aurait ressemblé17.»

Par cette curieuse formulation ( «voir sous la rue par en dessus »), le lecteur est invité à percevoir l'espace autour de lui comme illimité et véritablement tridimensionnel, comme le perçoit un oiseau dans le ciel ou comme les aviateurs doivent apprendre à l'appréhender afin de naviguer correctement, une compétence particulièrement nécessaire aux pilotes de chasse. Avant de devenir écrivain, Dahl s'était engagé dans la RAF de 1939 à 1942, participant notamment à la bataille d'Athènes et à la campagne de Syrie. Bien qu'il n'ait finalement été engagé en combat actif que pendant un mois, c'était un as, au sens militaire et aéronautique du terme, c'est-à-dire qu'il avait abattu au moins cinq appareils en combat aérien, et les témoignages sur sa formation de pilote attestent à la fois de ses capacités exceptionnelles et du bonheur intense qu'il éprouvait à voler ${ }^{18}$. Or, ce qui rend Mr Fox «fantastique» dans la version finale du roman, ce n'est pas son enthousiasme sans bornes, sa ténacité ou son optimisme contagieux. Il est rusé, certes, s'assurant par exemple toujours de se trouver sous le vent pour ne pas être repéré (chapitre 2), mais il s'agit là d'une ruse vieille comme le monde. Ce qui distingue Mr Fox des autres personnages, son superpouvoir pour ainsi dire, c'est sa capacité à se repérer dans l'espace grâce à la stratégie que les spécialistes des sciences cognitives appellent «la navigation à l'estime», sans s'appuyer sur des repères externes mais seulement sur lui-même pour retrouver son chemin, en intégrant ou en calculant continuellement la direction, la vitesse et l'effort physique dépensé pour aller d'un point à un autre. C'est un système qui est sujet à la dérive, puisque la moindre erreur peut prendre des proportions importantes, voire fatales 19 .

Dans la version publiée, même Blaireau se perd sous terre : «Cela fait trois jours et trois nuits que je creuse

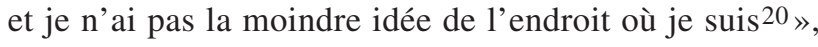

16. «And of course nobody on the street above had any idea of what was going on below them. », «Mr Fox », Maquette 1, sans pagination.

17. «If you had been able to see under the street from above, this is what it would have looked like.», «Mr Fox », Maquette 1, sans pagination.

18. Donald Sturrock, Storyteller: the Life of Roald Dahl, op. cit., p. $125-127$.

19. Amy E. Learmonth et Nora S. Newcombe, «The development of place learning in comparative perspective» dans Spatial cognition, spatial perception : mapping the self and space, F. L. Dolins et R. W. Mitchell (dir.), Cambridge, Cambridge UP, 2010, p. 522.

20. "I've been digging around in circles for three days and three nights and I haven't the foggiest idea where I am ! », Fantastic Mr Fox, chap. 12, p. 46. 
déclare-t-il à Mr Fox lorsque leurs galeries se croisent. À l'inverse, la réussite de Mr Fox, lorsque celui-ci localise le poulailler numéro 1 de Boggis dès la première tentative, est célébrée comme un exploit remarquable :

«J'ai réussi !», s'écria-t-il. «Du premier coup! J'ai réussi ! J'ai réussi !» Il se hissa par le trou dans le plancher et se mit à sauter et à danser de joie. [...] «Le poulailler Numéro Un de Boggis!», dit Mr Fox. «Exactement là où je voulais arriver! En plein dans le mille! Du premier coup! Vous ne trouvez pas ça fantastique? Et même génial, si je puis me permettre21 !»

Dans les deux premières maquettes de l'histoire, Mr Fox n'avait pas ce don. Le texte indique que le bruit des voitures et des camions circulant dans la grand-rue l'aide à se repérer, et on le voit vérifier où il se trouve visuellement en passant la tête par une bouche d'égout donnant à l'air libre avant de creuser la galerie latérale débouchant dans le supermarché. Dans la version finale en revanche, le personnage évolue au cours de l'histoire. Dans les premiers chapitres, Mr Fox s'appuie sur des indices visuels et olfactifs pour tenter de détecter le positionnement des fermiers, indices qui se révèlent insuffisants puisqu'il ne les voit pas à temps et perd sa queue. À la fin de l'histoire, il se montre capable d'utiliser la navigation à l' estime, c'est-à-dire à l'aveugle et se révèle être un véritable héros. Alors que Dahl avait suivi presque à la lettre les suggestions de son éditeur Fabio Coen, celui-ci n'avait pas mentionné ce don, qui n'apparaissait pas dans les deux maquettes originelles. C'est au fil des différentes réécritures, après la disparition des croquis dans les brouillons, que cette fascination de Dahl pour la représentation des déplacements dans un espace tridimensionnel est revenue, sous forme textuelle cette fois, par le biais des extraordinaires talents de navigateur de Mr Fox.

\section{Anthropomorphisme et altérité animale : le dessin comme révélateur}

Le passage par l'image oblige également l'auteur à se poser la question de l'anthropomorphisme des personnages, et rend visible ce questionnement pour le généticien. En effet, dans le tout premier brouillon, une note de régie au-dessus du croquis indique : «torches dans la gueule ?22 », un détail qu'une simple description textuelle n'aurait pas eu à régler. Le croquis représente les renards debout sur leurs pattes arrière, en train de pousser des caddies dans les galeries, portant dans leurs pattes avant des lampes d'où émergent des faisceaux de lumière, mais deux feuillets plus loin, les renards portent les torches dans la gueule, car ils courent maintenant à quatre pattes. Sur la page correspondante de la maquette, la phrase mentionnant les torches a été barrée au stylo-bille bleu : «Maintenant ils avaient des torches. Ils les avaient trouvées dans le supermarché. Les torches permettaient d'y voir facilement dans le tunnel23 » (voir fig. 6). Sur le croquis, on voit que Dahl a d'abord dessiné des torches dans les pattes des renards avant d'en gommer les faisceaux de lumière, les torches elles-mêmes étant plus difficiles à effacer sans abîmer le reste du dessin. De même, quelques pages plus loin, les torches avaient d'abord été dessinées dans la gueule des renards, puis gommées. Le statut des renards n'est pas clair, entre bi- et quadrupédie, capables d'y voir dans le noir mais tentés par l'emploi des outils que sont les torches et les caddies, désireux d'imiter le comportement des hommes, considérés comme supérieurs, tout en les évitant. Lorsque Dahl modifie son histoire et décide que les renards ne voleront plus de glacières et de tapis au supermarché, mais plutôt des poules chez les fermiers, ce n'est pas seulement une forme de moralité qui est restaurée, mais aussi l'identité des renards en tant qu'animaux non seulement en guerre contre les hommes, mais possédant des capacités étrangères à la nature humaine, comme celle de se repérer dans l'espace.

Si la proposition de Fabio Coen a été adoptée avec tant d'enthousiasme par Dahl, c'est bien parce qu'il s'est joué à ce moment-là quelque chose de décisif concernant la nature des personnages. Donner corps aux fermiers, antagonistes humains du héros, c'est par contraste placer au cœur de l'œuvre la radicale altérité des renards, voire de tous les

21. " "I've done it!" he yelled. "I've done it first time! I've done it! I've done it!" He pulled himself up through the gap in the floor and started prancing and dancing with joy.... "Boggis's Chicken House Number One !", cried Mr Fox. "It's exactly what I was aiming at! I hit it slap in the middle. First time! Isn't that fantastic ! And if I may say so, rather clever!"’, Fantastic Mr Fox, chap. 10, p. 40.

22. «flashlights in mouths?», «Mr Fox», premier brouillon (RD/2/9/1), fol. 28.

23. « Now they had flashlights. They had gotten them in the supermarket. The flashlights made it easy to see in the tunnel» «Mr Fox», Maquette 1, sans pagination. 
animaux. Or l'étude de l'avant-texte «Boggis and Bunce and Bean» révèle que la genèse de Fantastic Mr Fox est traversée par des réminiscences d'un autre texte de l'altérité animale, le roman édouardien de Kenneth Grahame The Wind in the Willows, un classique de la littérature enfantine britannique que Dahl connaissait bien ${ }^{24}$. En effet, la scène du premier brouillon où Mrs Fox emmène sa famille observer la maison coquette des hommes qui lui fait tant envie rappelle celle où Rat et Taupe, loin de chez eux, contemplent eux aussi avec nostalgie, depuis la rue et sans être vus, l'intérieur douillet des maisons d'un village (voir fig. 7 et 8 ). Le premier feuillet de la liasse du troisième brouillon n'est pas la page de titre mais comporte des griffonnages, listes de mots et bribes de phrases qui témoignent des réflexions de Dahl cherchant quels animaux inclure dans le «club des fouisseurs» («the Diggers'Club»), y compris des hermines, qui n'apparaîtront pas dans la version définitive (voir «stoat» au centre de la page, fig. 9). On lit au bas du feuillet une liste ( $«$ Mrs Fox $+3 /$ Mrs Badger + $3 /$ Mr \& Mrs Mole + 4/ Mr \& Mrs Rabbit + 5/ Mr \& Mrs Weasel $+3 » 25$ ) qui ressemble à une liste d'invités, sans doute pour le banquet final. On retrouve sur ce feuillet tous les animaux de The Wind in the Willows (excepté Crapaud, qui est d'ailleurs un personnage à part), qui n'apparaitront pas dans la version définitive. Ce banquet final rappelle celui qui clôt The Wind in the Willows, au manoir de Crapaud, après que celui-ci a été reconquis justement grâce à l'utilisation d'un passage secret souterrain.

Dans le chapitre «Dulce Domum», après avoir quitté le village où il avait contemplé avec Rat les intérieurs des maisons des hommes, Taupe sent soudainement la présence toute proche de sa propre maison et Grahame utilise cet épisode pour décrire la façon dont les animaux perçoivent le monde, d'une manière fondamentalement différente de la nôtre :

Nous autres les humains, qui avons perdu depuis longtemps le plus subtil des sens, nous n'avons pas de terme adéquat pour traduire les liens qu'entretiennent les animaux avec le monde qui les entoure, qu'il soit animé ou non; n'ayant que le mot flair pour désigner toute la gamme des frissons délicats qui excitent nuit et jour l'odorat des bêtes, soit pour les avertir, soit pour les stimuler, soit pour les éloigner 26 .

De même, d'abord dans les croquis des deux premières versions du roman, puis par son insistance sur les talents de

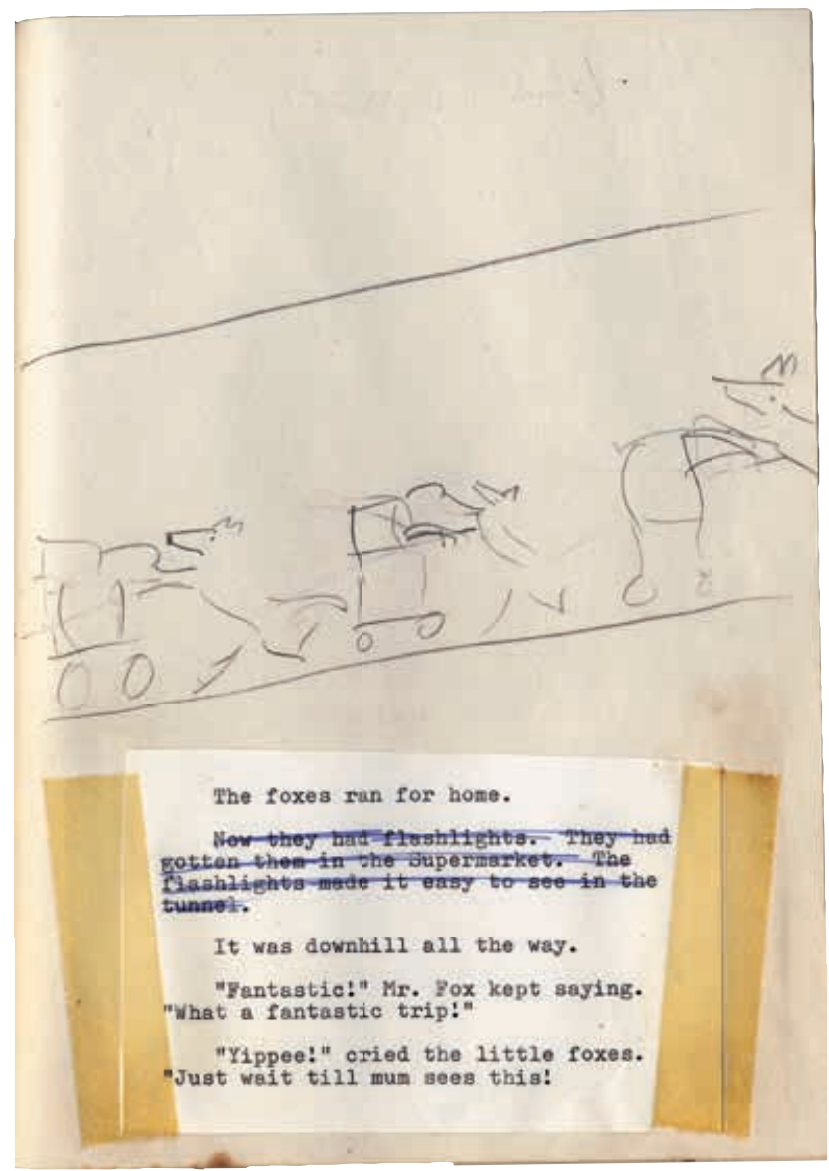

Fig. 6 : «The foxes ran for home», «Mr Fox», Maquette $1(\mathrm{RD} / 2 / 9 / 2)$, page de droite, pas de pagination.

(c) Roald Dahl Story Company Ltd

navigateur et la capacité à s'orienter de Mr Fox, Dahl semble tenter de donner à ressentir une expérience sensorielle du monde différente, voire de faire comprendre cette altérité animale. Ceci l'amène à poser une opposition finale assez étonnante entre le monde des hommes et la communauté des « diggers»: les «animaux qui creusent», une communauté qui rassemble des animaux traditionnellement ennemis dans la littérature enfantine, les renards et les lapins, pour qui

24. Échange privé avec Felicity Dahl. Mr Toad, Mr Rat et Mr Mole sont mentionnés dans la chanson improvisée par les Oompa-Loompas pour Mike Teavee (Charlie and the Chocolate Factory, chapitre 27).

25. «Boggis and Bunce and Bean», RD/2/9/6, fol. 1 .

26. Le Vent dans les saules, G. Joulié (trad.), Paris, Éditions Phébus, 2006, 81-82. «We others, who have long lost the more subtle of the physical senses, have not even proper terms to express an animal's inter-communications with his surroundings, living or otherwise, and have only the word «smell, » for instance, to include the whole range of delicate thrills which murmur in the nose of the animal night and day, summoning, warning, inciting, repelling. » 
96. Turs wrov ne rum vizLows

But it was from one little window, with its blind drawn down, a mere blank transpatency on the night, that the sense of bome and the litrle curnined world within walls - the barger stressfin world of outside Nature shut out and forgotten - most palsated. Close against the white blind hung a bird-cage, clearly sitbouetted, every wire, perch, and appurtenance distinct and recognizable, even to yesterday's dull-edged lamp of sugar. On the middle perch the fluffy oecripant, head tucked well into feathers, seemed so near

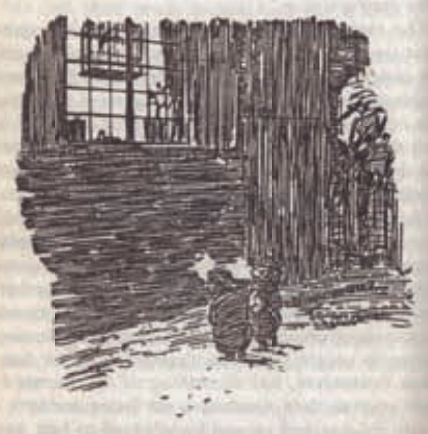

to them as to be easily strolked, had they tried; even the delicate tips of his plumped-out plumage pencilled

Fig. 7 : The Wind in the Willows, illustration d'Ernest H. Shepard, 1931.

Fig. 9 : «Boggis and Bunce and Bean», Brouillon 3 (RD/2/9/6), fol. 1. () Roald Dahl Story Company Ltd

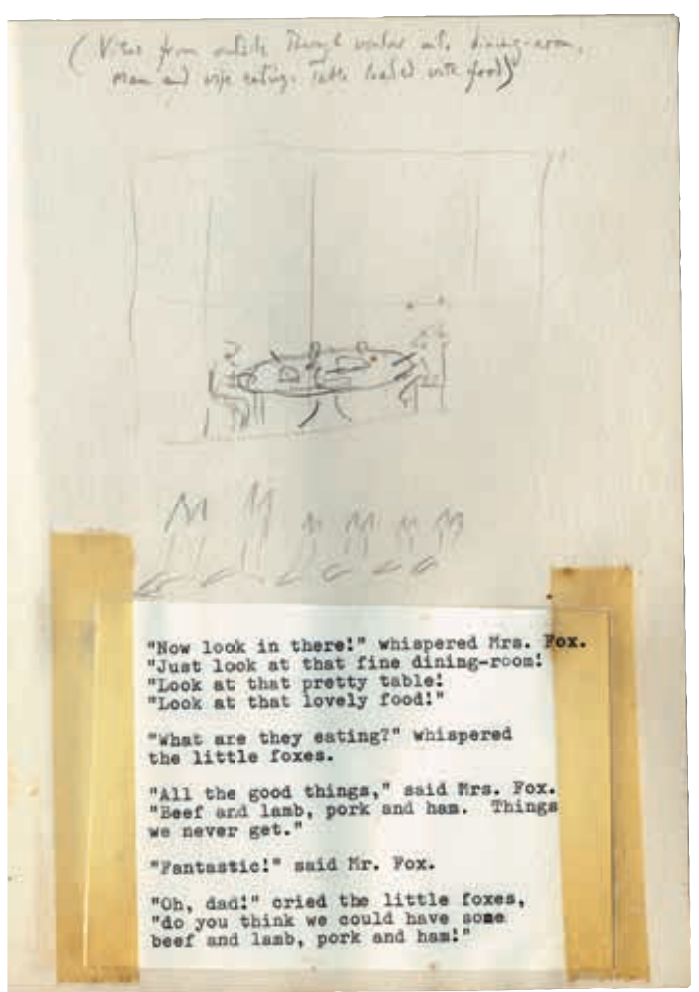

Fig. 8 : «"Now look in there!" whispered Mrs Fox.», «Mr Fox», Maquette 1 (RD/2/9/2), page de droite, pas de pagination. (c) Roald Dahl Story Company Ltd

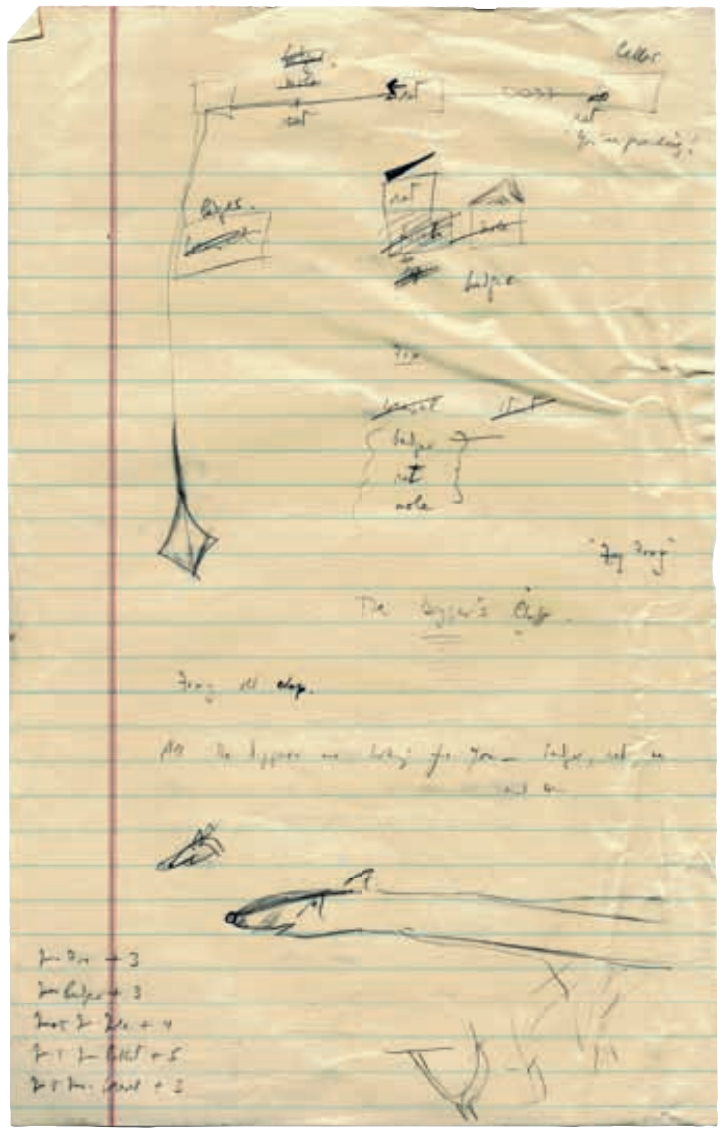


le plus jeune renardeau doit rappeler à son père de prendre des carottes (chap. 13). L'invention de cette catégorie taxinomique inhabituelle permet à Dahl de conserver l'opposition entre ses héros et les hommes tout en évitant l'écueil posé par les volailles dont tous se régalent. «Mais qui veut sortir à l'air libre, de toutes façons, je vous le demande? Nous sommes tous des fouisseurs, tous autant que nous sommes. Nous détestons l'air libre. Le monde d'en haut est plein d'ennemis 27 », déclare Mr Fox en guise de toast.

\section{Conclusion}

L'observation des avant-textes nous permet de voir Fantastic Mr Fox sous un nouveau jour et de prendre la mesure de l'importance de l'imagination visuelle et tridimensionnelle dans les processus créatifs de Dahl. Cet intérêt, ou cette sensibilité, pour les perceptions spatiales et sensorielles était également à l'œuvre dans The Gremlins, la toute première œuvre pour enfants de Dahl, publiée en 1943 alors qu'il n'avait publié par ailleurs qu'une seule nouvelle pour adultes, elle aussi puisée dans des souvenirs de pilote de guerre. On trouve dans The Gremlins des scènes très émouvantes, très fortes, en particulier la description d'un crash où l'on ressent avec le pilote la perte de tous les repères spatiaux, lorsque c'est le sol qui monte vers l'avion plutôt que l'avion qui tombe vers le sol. Le thème du réseau de galeries souterraines, voire de toute une ville souterraine, est présent aussi bien dans The Gremlins que dans Charlie and the Chocolate Factory avec le village des OompaLoompas, mais surtout aussi dans The Minpins (1991), la toute dernière œuvre de Dahl, qui, comme The Gremlins est un texte sur l'euphorie procurée par le vol et le pouvoir d'évoluer librement dans un espace à trois dimensions. Alors que Fantastic Mr Fox pouvait sembler appartenir à une autre veine, l'étude des brouillons tisse de nouveaux liens entre ce texte et le reste de l'œuvre de Dahl et ouvre de nouvelles possibilités d'interprétation.

La carrière de pilote de Dahl s'arrêta en 1941 lorsque les conséquences d'un crash l'empêchèrent de continuer à voler et le conduisirent à devenir écrivain, d'abord au service de propagande de la RAF pour raconter ses souvenirs de vaillant combattant, mais aussi parce que, selon lui, le coup qu'il avait reçu à la tête avait révélé ses talents d'écrivain 28 . Lui-même avait comparé sa hutte d'écrivain 29 à un cockpit, car il y travaillait dans un fauteuil exactement à sa taille, posait un plan de travail ajusté exactement à ses besoins sur les accoudoirs et casait ses longues jambes dans un sac de couchage, si bien qu'une fois installé il ne pouvait plus bouger. Les suites de cet accident lui avaient fait perdre la possibilité de revoler, mais il en avait retrouvé le bonheur dans l'écriture qu'il décrit comme une promenade dans un espace en trois dimensions où les points de vue changent sans cesse :

Quand on écrit, c'est comme partir pour une longue randonnée à travers des vallées et des montagnes : on arrive au premier point de vue, et on l'écrit, puis on marche un peu plus loin, peut-être jusqu'en haut d'une colline, et on voit autre chose et on écrit ça, et ainsi de suite 30 .

27. « But who wants to go out, anyway; let me ask you that? We are all diggers, every one of us. We hate the outside. The outside is full of enemies. » Fantastic Mr Fox, chap. 17, p. 79.

28. «A monumental bash on the head», entretien avec Peter Lennon, The Times, 12 décembre 1983, cité dans D. Sturrock, op. cit., p. 135.

29. Cf. photo p. 113, fig. 3 du présent numéro.

30. «When you're writing it's rather like going on a very long walk, across valleys and mountains and things, and you get the first view of what you see and you write it down. Then you walk a bit further, maybe on top of a hill, and you see something else, then you write that and you go on like that », cité dans Frances E. Ruffin, Meet Roald Dahl, New York, Powerkids Press, 2006, non paginé. 
Christine Collière-Whiteside est maître de conférences à l'ESPE de Bourgogne, Université de Bourgogne-Franche Comté. Ses travaux portent sur les genèses des œuvres de Lewis Carroll, George McDonald et Roald Dahl. Elle travaille également sur la didactique de l'écriture et de la réécriture. Elle a co-dirigé Apprendre de l'intime : entre littérature et langues, EME, 2016 et co-organisé deux journées d'études sur les brouillons d'écoliers et les brouillons d'écrivains à l'ESPE de Mâcon (2016) et à l'ESPE de Lille (2017).

christine.colliere-whiteside@u-bourgogne.fr

\section{Roald Dahl auteur-illustrateur : de l'image au texte, les débuts de la genèse de Fantastic Mr Fox}

Le dossier génétique de Fantastic Mr Fox, conservé aux archives Roald Dahl à Great Missenden, présente le début de genèse de deux maquettes fabriquées par Dahl, occurrence unique dans les archives d'un travail simultané de l'auteur sur le texte et les illustrations. L'étude génétique du dossier fait émerger l'importance de l'imagination visuelle dans la conception de l'histoire et des personnages, avant toute collaboration avec un illustrateur, et en particulier d'une perception tridimensionnelle du monde, que nous proposons de lire à la lumière du passé de pilote de l'auteur. L'analyse des avant-textes montre l'auteur s'interrogeant sur le degré d'anthropomorphisme de ses personnages, la représentation de leur expérience sensorielle du monde et la défamiliarisation que cela entraîne et permet de faire émerger une intertextualité insoupçonnée entre Fantastic Mr Fox et The Wind in the Willows (K. Grahame) et d'autres textes de Dahl tels que The Gremlins ou The Minpins.

The genetic documents for Fantastic Mr Fox, kept in the Roald Dahl Archives in Great Missenden, contain two illustrated notebooks on which the author worked simultaneously on text and illustrations, a unique occurrence in the Dahl files. The genetic study of the documents reveals the importance of visual imagination in the conception of the story and the characters, before any collaboration with an illustrator, and shows the importance of Dahl's three-dimensional perception of the world, which can be read in the light of Dahl's past experiences as a pilot. The analysis of those drafts shows that the author had to solve problems linked to the degree of anthropomorphism of the characters, the representation of their sensory experience of the world and the defamiliarisation that it entails. It also allows us to discover some new intertextual relationships between Fantastic Mr Fox and Kenneth Grahame's The Wind in the Willows as well as with some of Dahl's texts, such as The Gremlins or The Minpins.

Das genetische Dossier zu Fantastic Mr Fox, die in den „, RoaldDahl-Archiven" in Great Missenden aufbewahrt werden, enthalten zwei illustrierte Notizbücher, an denen der Autor zugleich im Hinblick auf Text und Illustrationen arbeitete, was einen einmaligen Fall im Bereich der Dahl-Bestände darstellt. Das genetische Studium der Dokumente enthüllt die Bedeutung der visuellen Imagination in der Konzeption der Geschichte und ihrer Charaktere - noch vor der Zusammenarbeit mit einem Illustrator - und zeigt, eine wie große Rolle die dreidimensionale Anschauung bei Dahl spielte. Dies kann im Lichte von Dahls früheren Erfahrungen als Pilot gesehen werden. Die Analyse dieser avant-textes zeigt, dass der Autor Probleme zu lösen hatte, die mit dem Grad des Anthropomorphismus der Charaktere zusammenhängen, sowie mit der Darstellung ihrer sinnlichen Welterfahrung und der damit einhergehenden Entfremdung. Dies erlaubt uns, neue intertextuelle Beziehungen zwischen Fantastic Mr Fox und Kenneth Grahames
The Wind in the Willows zu entdecken, aber auch zu einigen von Dahls anderen Texten wie etwa The Gremlins oder The Minpins.

E1 dossier genético de Fantastic Mr Fox, conservados en los archivos Roald Dahl en Great Missenden, presenta el comienzo de la génesis de dos maquetas fabricadas por Dahl, único caso en los archivos de un trabajo simultáneo del autor con el texto y las ilustraciones. El estudio genético del dossier revela la importancia de la imaginación visual en la concepción de la historia y de los personajes, previa a toda colaboración con el ilustrador, y, en particular, una concepción tridimensional del mundo que puede ser leída a la luz del pasado de piloto del autor. El análisis de los pre-textos pone de manifiesto los interrogantes del autor acerca del grado de antropomorfismo de sus personajes, de la representación de su experiencia sensorial del mundo y de la consiguiente desfamiliarización, y permite revelar una intertextualidad insospechada entre Fantastic Mr Fox, The Wind in the Willows de K. Grahame y otros textos de Dahl, como The Gremlins o The Minpins.

$\mathbf{O}$ dossier genético de Fantastic Mr Fox, guardado no arquivo Roald Dahl em Great Missenden, revela o início da gênese de duas maquetes produzidas por Dahl, ocorrência única no arquivo de trabalho simultâneo do autor conduzido sobre o texto e sobre as ilustrações. Sobressai a importância da imaginação visual na concepção da história e das personagens, antes de qualquer colaboração com um ilustrador, tirando partido da percepção tridimensional que Dahl tinha do mundo, graças à sua experiência como piloto. A análise desses esboços mostra o autor a tentar resolver problemas postos pelo grau de antropomorfismo dos personagens, como representar a sua experiência sensorial e a sua estranheza perante o mundo. De tudo isso emerge uma inesperada relação intertextual entre Fantastic Mr Fox e The Wind in the Willows (K. Grahame), e ainda outros textos de Dahl como The Gremlins ou The Minpins.

I 1 dossier genetico di Fantastic Mr Fox, conservato negli archivi Roald Dahl a Great Missenden, contiene due quaderni illustrati che rappresentano un caso unico di lavoro simultaneo sul testo e sulle immagini. Lo studio genetico del dossier mette in luce l'importanza dell'immaginazione visiva nella concezione della storia e dei personaggi, prima di qualsiasi collaborazione con un illustratore, e rivela in particolare una percezione tridimensionale del mondo, che proponiamo di interpretare alla luce del passato di pilota dell'autore. L'analisi degli avantesti mostra le interrogazioni dell'autore sul grado di antropomorfismo dei suoi personaggi, la rappresentazione della loro esperienza sensoriale del mondo e la "defamiliarizzazione" conseguente, permettendo - inoltre - di far emergere un'intertestualità inaspettata tra Fantastic Mr Fox e The Wind in the Willows di K. Grahame, o altri testi di Dahl, quali The Gremlins o The Minpins. 